\title{
A MULTI-WAVELENGTH VIEW OF GALAXY EVOLUTION WITH AKARI
}

\author{
S. Serjeant ${ }^{1}$, C. Pearson ${ }^{1,2}$, G. J. White ${ }^{1,2}$, M. W. L. Smith ${ }^{3}$, And Y. Doi ${ }^{4}$ \\ ${ }^{1}$ Department of Physical Sciences, The Open University, Milton Keynes, MK7 6AA, UK \\ ${ }^{2}$ RAL Space, STFC Rutherford Appleton Laboratory, Chilton, Didcot, Oxfordshire, OX11 0QX, UK \\ ${ }^{3}$ School of Physics and Astronomy, Cardiff University, Queens Buildings, The Parade, Cardiff CF24 3AA, UK \\ ${ }^{4}$ Department of General System Studies, Graduate School of Arts and Sciences, The University of Tokyo, 3-8-1 \\ Komaba, Meguro-ku, Tokyo 153-8902, Japan \\ (Received July 20, 2012; Accepted August 17, 2012)
}

\begin{abstract}
AKARI's all-sky survey resolves the far-infrared emission in many thousands of nearby galaxies, providing essential local benchmarks against which the evolution of high-redshift populations can be measured. This review presents some recent results in the resolved galaxy populations, covering some well-known nearby targets, as well as samples from major legacy surveys such as the Herschel Reference Survey and the JCMT Nearby Galaxies Survey. This review also discusses the prospects for higher redshifts surveys, including strong gravitational lens clusters and the AKARI NEP field.
\end{abstract}

Key words: infrared: telescope; conferences: proceedings

\section{INTRODUCTION}

This paper will review results on bright galaxies from AKARI and Herschel, and will cover from local galaxies to high-z galaxies, via gravitational lensing.

The first part of the review will be about the AKARI XTENDED Prioritised Study (PS) program. We have heard a great deal about the AKARI diffuse maps so far in this conference and this paper will focus on some early results on resolved galaxies. We will cover a few obvious old friends, such as Andromeda, and then progress from these anecdotal examples to discussing results from two major legacy surveys with robust selection criteria. Note that no claim will be made that any of these surveys are unbiased in any sense. There is no such thing as an unbiased survey in astronomy. A 'bias' is just a pejorative way of referring to the selection effects, and any astronomical catalogue of any nature has selection criteria of some sort. (Even "all luminous objects within this volume" would neglect neutral hydrogen systems, and would in practice have a luminosity limit anyway; astronomical surveys are defined by what they exclude, not what they include.) Instead of trying to perform the impossible feat of avoiding 'biases', one must understand and quantify one's selection effects, which is the key advantage of these surveys over heterogenous compilations.

Having described some of the early results from the XTENDED PS program, the focus will move onto the Herschel ATLAS survey. This is in some ways a complementary project to the AKARI all-sky survey, and has mapped $1 \%$ of the sky to almost as shallow a depth as Herschel can achieve. Herschel ATLAS covers local as well as high redshift galaxies, and we'll go from low redshift to high redshift via gravitational lensing. Staying on the lensing theme, this review will discuss recent evidence for the links between the Herschel and AKARI populations that have been found using the cluster lens Abell 2218. Finally, this paper reviews the prospects for Herschel data in the AKARI NEP-Deep field, in the light of the current results from Herschel ATLAS and other surveys. 

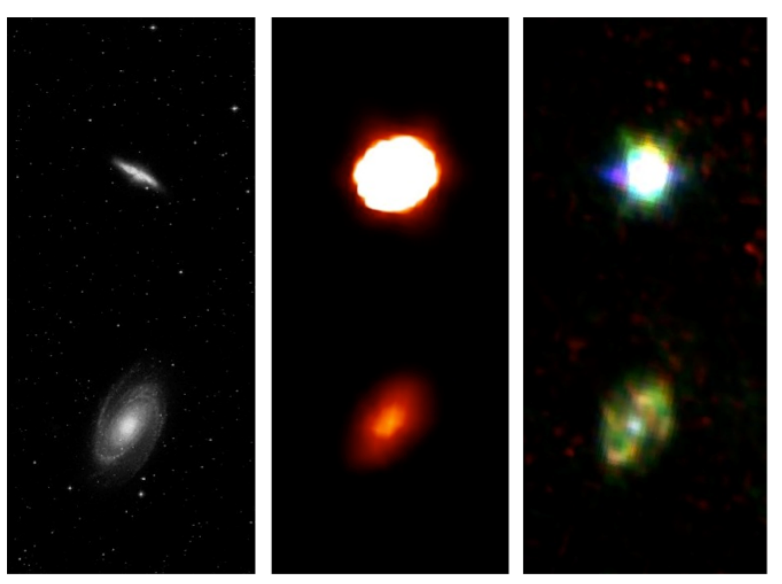

Fig. 1. M82 (top) and M81 (bottom) in DSS B-band (left), IRAS $60 \mu \mathrm{m}$ (centre) and in the AKARI all-sky survey (right, with $160,140,90 \mu \mathrm{m}$ as RGB respectively). Note the arms in M81 resolved by AKARI. The two galaxies are separated by about $37^{\prime}$, or $(38 \pm 5) \mathrm{kpc}$ in projection.

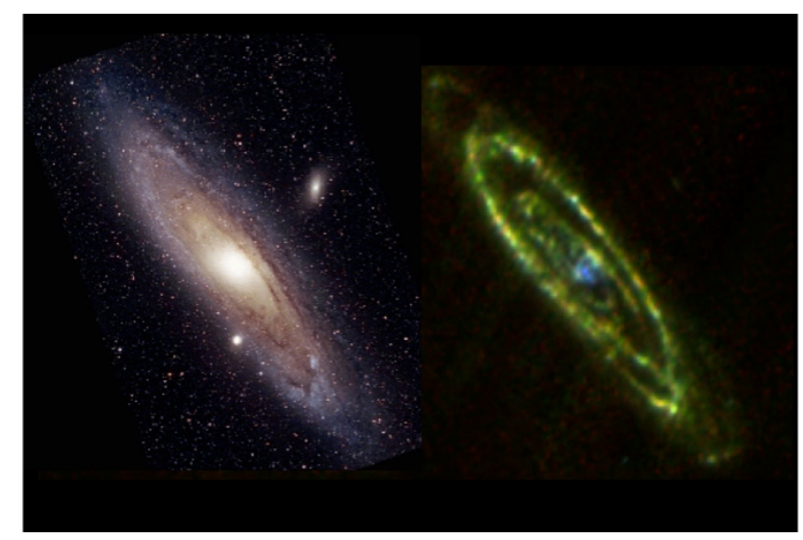

Fig. 2. M31 in the optical (left; NASA APOD, Jason Ware) and in the AKARI all-sky survey (right, with $160,140,90 \mu \mathrm{m}$ as RGB respectively). The diameter of M31 is approximately $30 \mathrm{kpc}$, and $1^{\circ} \equiv 13.6 \mathrm{kpc}$. Note the star-forming ring $\sim 10 \mathrm{kpc}$ from the centre, and the warm central dust (also associated with a $\mathrm{CO}$ deficit).

\section{THE AKARI XTENDED PRIORITISED STUDY}

The goal of the XTENDED program is the scientific exploitation of an all-sky far-infrared atlas of resolved galaxies from AKARI. Unlike, e.g., the heterogenous SINGS survey in which the selection criteria are difficult to quantify (Kennicutt et al., 2003), the sam- ple is limited purely by far-infrared surface brightness. The project will test the frequency of occurence of cool extended dust components undetectable by IRAS, construct spatially-resolved radio/far-infrared correlations, map every known blue compact dwarf galaxy, and help determine the extent to which integrated properties of galaxies are reliable measures of the mean physical conditions within them. Work on the whole sample is still ongoing so this paper will discuss only a few key targets. There are rich seams to be mined. Figure 1 compares AKARI diffuse maps and IRAS images of the prototypical starburst M82, plus its companion M81. What is immediately clear is the improved angular resolution of AKARI, with the arms of M81 clearly discernable. AKARI succeeds in resolving the dust emission in many thousands of local galaxies.

Figure 2 shows another old friend, M31, at optical and far-infrared wavelengths. The morphology agrees with ISO and Spitzer far-infrared observations (Haas et al., 1998; Gordon et al., 2006). M31 has the interesting property that $90 \%$ of the far-infrared emission is not associated with star formation. There is a higher temperature knot in the cold dust distribution at the centre of M31, associated with a deficit in $\mathrm{CO}$, and there is also a star-forming ring about $10 \mathrm{kpc}$ from the centre. The diameter of M31 is about $30 \mathrm{kpc}$ in this image.

Figure 3 presents optical and far-infrared data on NGC 253, which has far-infrared emission from a superwind driven by its starburst. However note the yellow pixel in the centre, caused by saturation; clearly, care needs to be taken with far-infrared photometry of bright galaxies in the AKARI all-sky survey.

As a sanity check of the diffuse map fluxes, large aperture photometry measurements $\left(4.75^{\prime}\right.$ radius) were taken of Arp 220 in the all-sky diffuse maps. The flux measurements were $72,74,51,68 \mathrm{Jy}$ (all $\pm 1 \mathrm{Jy}$ ) in the filters N60, WIDE-S, WIDE-L and N160 respectively, i.e. at $65 \mu \mathrm{m}, 90 \mu \mathrm{m}, 140 \mu \mathrm{m}$ and $160 \mu \mathrm{m}$ respectively. These are up to a factor of two fainter than catalogued fluxes from ISO and IRAS, which we believe is due to detector saturation in AKARI and/or incorrect flagging of peak fluxes as glitches (see e.g. Yamamura et al., 2010), although no saturation features such as that in Figure 3 were obviously visible in the images in any band. As a result we will restrict our discussion to targets with predicted fluxes $<50$ Jy per beam. 


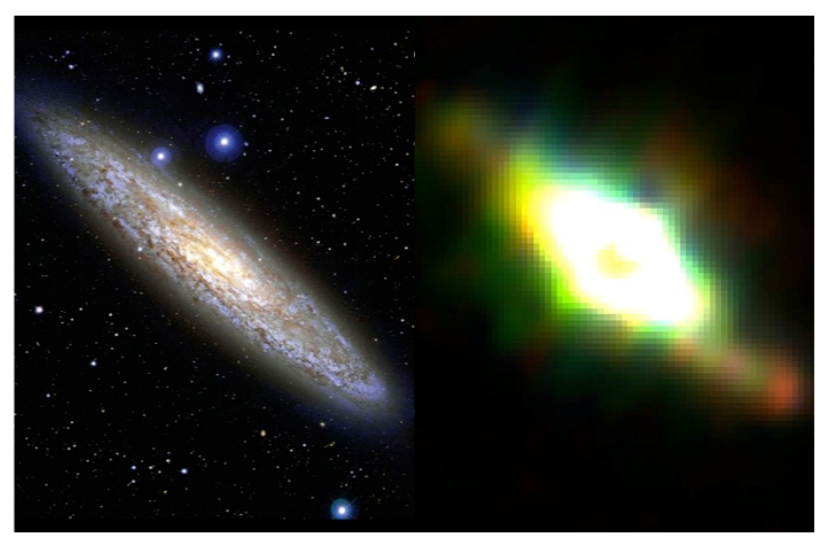

Fig. 3. Optical image of NGC 253 (left; NASA APOD, CFHT) compared to the AKARI all-sky survey image (right) with 160,140,90 $\mu \mathrm{m}$ rendered as RGB respectively. The optical diameter is $27.5^{\prime} \equiv(28 \pm 2) \mathrm{kpc}$. Note the superwind and the central saturated pixel.

\section{AKARI OBSERVATIONS OF THE HERSCHEL REFERENCE SURVEY}

So far we have covered only a few anecdotal examples of well-known galaxies. There is only so far one can go with anecdotal evidence, so the next stage is to move to large samples with well-understood selection criteria. The first sample we will discuss is the Herschel Reference Survey (Boselli et al., 2010). This is a guaranteed time programme on Herschel to map about 300 local galaxies. It is volume limited and K-band limited, implying an effective minimum stellar mass selection depending on distance. It has many science goals, including a census of dust along the Hubble sequence, the connections between star formation and dust emission, the global extinction in galaxies as a function of type, the presence of dust in ellipticals and the cycle of dust destruction and creation. Ciesla et al. (2012) present submm data for galaxies in this sample. The survey only obtained submm Herschel data with the SPIRE instrument, and as a result over a third of their targets lack far-infrared data entirely. The AKARI all-sky survey is ideal to address this need.

As part of the XTENDED PS program, fluxes for the Herschel Reference Survey were extracted in a $300^{\prime \prime} \times 300^{\prime \prime}$ box around each galaxy. Sky subtraction was achieved by iteratively estimating the mode of the pixel flux histogram in the $\left(1.5^{\circ}\right)^{2}$ postage stamps around each target, rejecting $>3 \sigma$ or $<-3 \sigma$ outliers.
Flux errors were estimated by convolving the postage stamp with a kernel equivalent to the photometric aperture, then iteratively measuring the variance of the pixel count histogram of the smoothed image.

Careful sky subtraction was found to be important for the photometry, but there are still unresolved problems. Figure 4 shows two example SEDs from the combined Herschel and AKARI data from the Herschel Reference Survey. Clearly at least in the case of NGC 4100 (and in other targets not shown), there are still unresolved systematics in the fluxes; the discrepancies with the models suggest the systematics are no more than $\sim 30 \%$. With this caveat in mind, we estimated dust masses assuming single temperature grey-body fits (see e.g. Dunne et al., 2011). Clearly, galaxies do not have single temperatures, or even a few discrete temperatures; this is very much a "spherical cow" approximation. In some cases, an excess over the single-temperature fits at shorter AKARI wavelengths requires the existence of warmer dust phases. Future work will make use of radiative transfer modelling. For the present purposes, the fits are used only to provide order of magnitude estimates for dust masses, which will in any case be dominated by the cooler components. The grey body fitting assumed an emissivity of $\beta=1.5$. Dust mass estimates are typically a few $\times 10^{7} M_{\odot}$ in this sample. Work is ongoing to improve the photometry in this sample.

\section{AKARI OBSERVATIONS OF THE JCMT NEARBY GALAXIES SURVEY}

The JCMT Nearby Galaxies Survey (Wilson et al., 2012) is another major legacy survey, with the goal of resolving sub-kpc structure in over 150 nearby galaxies. The goals, amongst others, are to see how morphology and environment affect star formation in nearby galaxies. The survey is covering the Spitzer SINGS sample (Kennicutt et al., 2003), in order to benefit from the multi-wavelength legacy data in SINGS. However, SINGS has a somewhat heterogeneous selection, so the JCMT survey is also observing neutral-hydrogenselected samples in the field and in Virgo. Again, the trouble with robust statistical selection is that one is often left with not much supplementary data. In particular, the field sample lacks far-infrared data. As with the Herschel Reference Survey, the AKARI diffuse maps can provide this far-infared data, sampling the peaks of the bolometric outputs of the galaxies. 

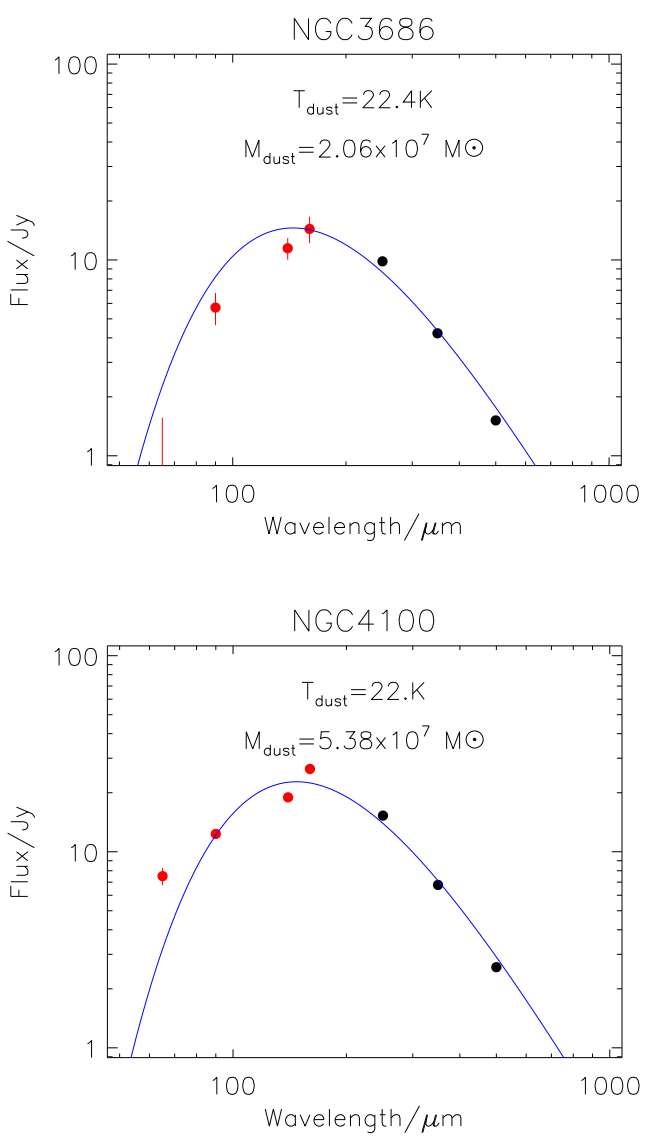

Fig. 4. SEDs of two galaxies in the Herschel Reference Survey. The $>200 \mu \mathrm{m}$ data is from Herschel SPIRE, while $<200 \mu \mathrm{m}$ is from AKARI. The blue line is a grey body fit assuming an emissivity of $\beta=1.5$. Note that the AKARI data clearly still has sources of systematic error of the order of $\sim 30 \%$ of unknown origin, but possibly related to sky subtraction.

Figure 5 shows an example target from this survey, seen in the AKARI all-sky survey. Note that AKARI's angular resolution has the important advantage over IRAS that it can decouple flux from the galaxy from foreground cirrus structure.

Fluxes at $90 \mu \mathrm{m}$ (WIDE-S filter) were extracted for all the JCMT nearby galaxies field sample following the same procedure as for the Herschel Reference Survey above, safely encompassing the optical diameters of the galaxies. Figure 6 shows the $\mathrm{CO}(3-2)$ luminosities compared to the far-infrared luminosities of homogenouslyselected high-redshift galaxies from Iono et al. (2009), plus the AKARI measurements of the JCMT sample. The line is the extrapolation from the high-redshift

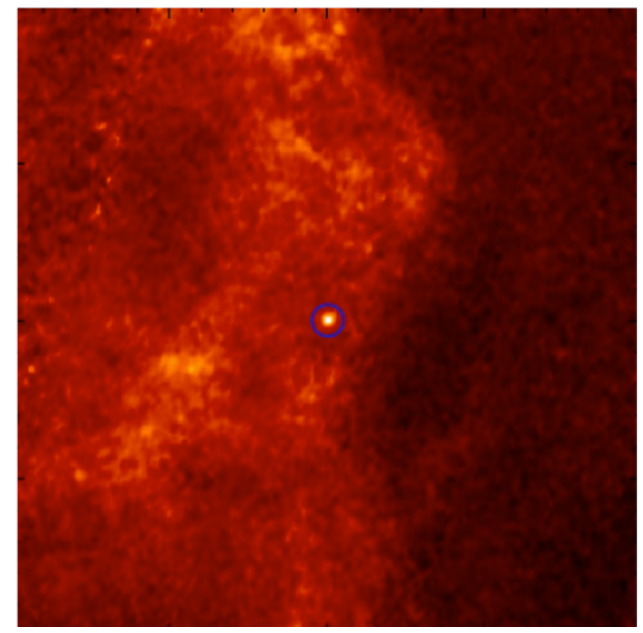

Fig. 5. AKARI all-sky $90 \mu \mathrm{m}$ image of NGC 1156, from the JCMT Nearby Galaxies Survey. Note the surrounding cirrus structure. Lower angular resolution data would have difficulty decoupling the foreground cirrus and background galaxy. The image is $1.5^{\circ}$ on a side.

populations, and is not a fit to the JCMT data. This extends the Iono et al. relation over five orders of magnitude in homogenously-selected samples. The overall relation is not quite linear, implying that the JCMT sample have far-IR to CO ratios lower than the ULIRGs in general. One can convert the far-infrared to $\mathrm{CO}$ luminosity ratio into a star formation rate to molecular gas mass ratio, resulting in a timescale. From this, one can derive a molecular gas depletion timescale of about 3 Gyr in the JCMT sample. The gas depletion timescale in submm galaxies is more like $0.5 \mathrm{Gyr}$, once one has remembered that different $\mathrm{CO}$ to $\mathrm{H}_{2}$ conversions are appropriate for ultraluminous infrared galaxies and submm galaxies.

\section{LOCAL GALAXIES AND LENSES IN HER- SCHEL ATLAS}

The Herschel Astrophysical Terahertz Large Area Survey (H-ATLAS, Eales et al., 2010) is perhaps Herschel's answer to the AKARI all-sky survey. It has mapped about $1 \%$ of the sky to almost the submm confusion limits, discovering NGC 4725 to have an "Andromeda analogue" dust ring. Many hundreds of thousands of local galaxies are expected in the survey; see e.g. Baes et al. (2010) for an early example of a nearby galaxy with components of highly obscured star formation which nonetheless contribute little to the global extinction in the galaxy. AKARI detections of Herschel ATLAS local 


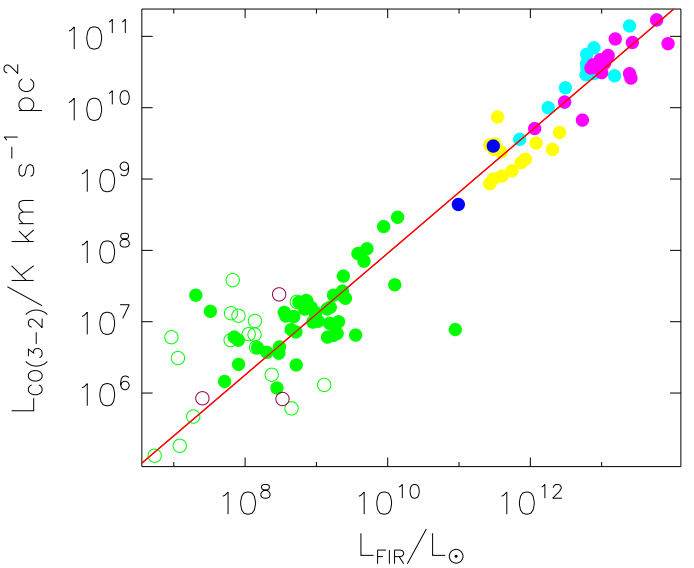

Fig. 6. $\mathrm{CO}(3-2)$ vs. far-infrared luminosity-luminosity correlation for the homogenously-selected Iono et al. (2009) data (luminous infrared galaxies: yellow, submm-selected galaxies: cyan, quasars: magenta, Lyman-break galaxies: blue), plus the AKARI $90 \mu \mathrm{m}$ data on the JCMT nearby galaxies (spirals: green filled circles, irregulars: green open circles, others: brown open circles). The red power-law line is not quite a linear relation, and is an extrapolation from the Iono sample, not a fit to the JCMT data. Photometric errors in the JCMT sample are not shown for clarity but are consistent with the scatter.

galaxies are discussed at this conference in Pearson et al. (this volume). In roughly equal numbers at $500 \mu \mathrm{m}$ to local galaxies, H-ATLAS also made the landmark discovery of a large population of strong gravitational lenses (Negrello et al., 2010), ultimately caused by the steep intrinsic $500 \mu \mathrm{m}$ bright number counts of highredshift galaxies. There are many examples of submm galaxies with $z>2$ far-infrared photometric redshifts but identifications with foreground spirals or ellipticals; not every obvious local galaxy optical ID is the site of the observed far-infrared emission. HST, IRAM, Herschel, SMA and other follow-ups are all ongoing.

\section{AKARI DEEP FIELDS: LINKING THE LOCAL AND HIGH-REDSHIFT UNIVERSE}

A further strong gravitational lensing system, studied by both AKARI and Herschel, is the galaxy cluster Abell 2218. At the 2009 AKARI conference we had just extended the $15 \mu \mathrm{m}$ galaxy source counts an order

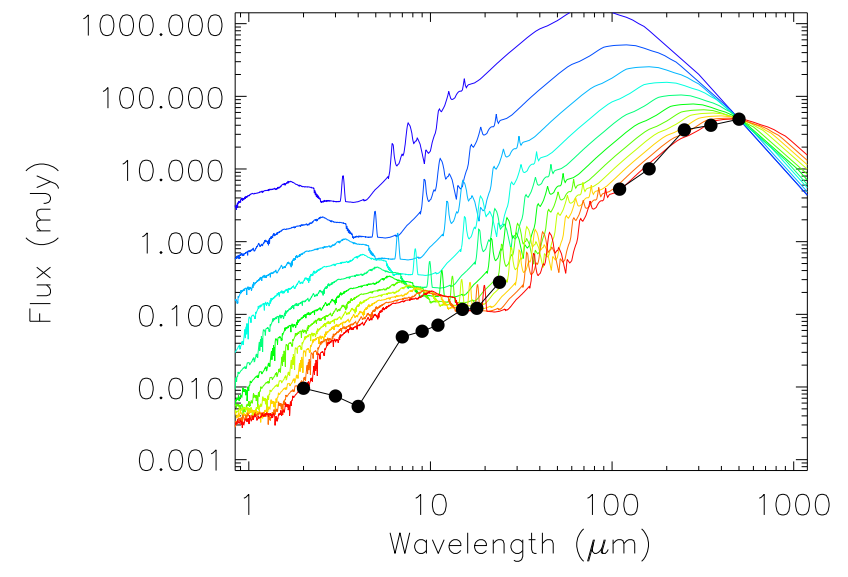

Fig. 7. M82 local template, normalised to the $500 \mu \mathrm{m}$ confusion limit, from $z=0$ increasing in steps of $\delta z=0.5$ (blue to red). Also shown are the SPIRE confusion limits, the NEP-Deep AKARI depths, and the PACS depths assuming all priority 2 scheduled data is obtained. Note that the AKARI depths are more than sufficient to detect the submm-selected population.

of magnitude fainter than any other surveys, exploiting the lensing magnifications (Hopwood et al., 2010). Since then, Hopwood et al. (in prep.) have performed bespoke analysis of the SPIRE submm data in the field, stacked the submm fluxes of the AKARI $15 \mu \mathrm{m}$-selected population, and found the $15 \mu \mathrm{m}$ population is responsible for $\sim 40-30 \%$ of the FIRAS backgrounds at $250-500 \mu \mathrm{m}$, with the uncertainties dominated by the $5 \sigma$ infrared background measurements.

It should not be altogether surprising that there are such strong links between the mid-infrared and submm populations. To demonstrate this, we return finally to the first local galaxy discussed in this paper, M82. Figure 7 shows redshifted M82 template SEDs normalised to the $500 \mu \mathrm{m}$ confusion limit compared to the AKARI NEP-Deep mid-infrared depths. The optical stellar populations will obviously not be representative of the high-redshift population but the midinfrared fluxes should be realistic. The AKARI depths are clearly easily deep enough to detect the the $500 \mu \mathrm{m}$ population with this SED.

The NEP field has already been observed with Herschel in a $9 \mathrm{deg}^{2}$ map, with more data still scheduled in 
priority 2 time. SCUBA-2 and LOFAR data are also imminent, and the field has been the target of many other multi-wavelength campaigns. In the longer term, the Euclid mission (launch, 2019) will, in addition to its $20,000 \mathrm{deg}^{2}$ wide survey, devote $40 \mathrm{deg}^{2}$ to deep cosmological fields. The location or locations of these $40 \mathrm{deg}^{2}$ are determined partly by the need to cover famous fields with lots of multi-wavelength legacy data, and partly by the constraints of the scanning strategy of the mission. The latter in particular very strongly favours the ecliptic poles, which are the natural deep field locations for many space observatory and survey missions.

\section{CONCLUSIONS}

Preliminary photometry for galaxies in the Herschel Reference Survey demonstrates dust masses typically a few $\times 10^{7} M_{\odot}$. These would be unmeasurable without the AKARI all-sky survey. However, systematic errors of unknown origin of the order $\sim 30 \%$ are still present in at least some photometric measurements; work is ongoing to improve this photometry. Preliminary photometry for galaxies in the JCMT Nearby Galaxies Survey extends the far-infrared: $\mathrm{CO}(3-2)$ luminosityluminosity correlation down five orders of magnitude in homogenously-selected samples. The molecular gas depletion timescale for JCMT Nearby Galaxies Survey targets is typically $\sim 3 \mathrm{Gyr}$, about an order of magnitude longer than in high-redshift submm-selected galaxies. The AKARI ultra-deep $15 \mu \mathrm{m}$ population contributes about a third or more of the extragalactic background light at $250-500 \mu \mathrm{m}$.

\section{ACKNOWLEDGEMENTS}

We thank the anonymous referee for helpful comments. SS thanks STFC for financial support under grants ST/G002533/1 and ST/J001597/1. The Second AKARI Conference was supported by BK21 program to Seoul National University by the Ministry of Education, Science and Technology, Center for Evolution and Origin of Universe (CEOU) at Seoul National University, National Research Foundation Grant No. 2006341-C00018 to HMLee, Astronomy Program, Seoul National University Nagoya University Global COE Program: Quest for Fundamental Principles in the Universe, Division of Particle and Astrophysical Science, Nagoya University, and Institute of Space and Astronautical Science, Japan Aerospace Exploration Agency. This research has made use of the NASA/IPAC Extra- galactic Database (NED) which is operated by the Jet Propulsion Laboratory, California Institute of Technology, under contract with the National Aeronautics and Space Administration.

\section{REFERENCES}

Baes, M., et al., 2010, Herschel-ATLAS: The Dust Energy Balance in the Edge-on Spiral Galaxy UGC 4754, A\&A, 518, L39

Boselli, A., et al., 2010, The Herschel Reference Survey, PASP, 122, 261

Ciesla, L., et al., 2012, Submillimetre Photometry of 323 Nearby Galaxies from the Herschel Reference Survey, A\&A in press (arXiv:1204.4726)

Dunne, L., et al., 2011, Herschel-ATLAS: Rapid Evolution of Dust in Galaxies Over the Last 5 Billion Years, MNRAS, 417, 510

Eales, S. A., et al., 2010, The Herschel ATLAS, PASP, 122,499

Gordon, K. D., et al., 2006, Spitzer MIPS Infrared Imaging of M31: Further Evidence for a SpiralRing Composite Structure, ApJL, 638, 87

Haas, M., et al., 1998, Cold Dust in the Andromeda Galaxy Mapped by ISO, A\&A, 338, L33

Hopwood, R., et al., 2010, Ultra Deep Akari Observations of Abell 2218: Resolving the $15 \mu \mathrm{m}$ Extragalactic Background Light, ApJL, 716, 45

Iono, D., et al., 2009, Luminous Infrared Galaxies with the Submillimeter Array. II. Comparing the CO (3-2) Sizes and Luminosities of Local and HighRedshift Luminous Infrared Galaxies, ApJ, 695, 1537

Kennicutt, R. C., et al., 2003, SINGS: The SIRTF Nearby Galaxies Survey, PASP, 115, 928

Negrello, M., et al., 2010, The Detection of a Population of Submillimeter-Bright, Strongly Lensed Galaxies, Science, 330, 800

Wilson, C. D., et al., 2012, The JCMT Nearby Galaxies Legacy Survey - VIII. CO Data and the $L_{C O(3-2)}-L_{\mathrm{FIR}}$ correlation in the SINGS sample, MNRAS in press (arXiv:1206.1629)

Yamamura, I., et al., 2010, AKARI/FIS All-Sky Survey Bright Source Catalogue Version 1.0 Release Note 